\title{
Robust Resonant Frequency-Based Contact Detection With Applications in Robotic Reaching and Grasping
}

\author{
Spencer B. Backus, Student Member, IEEE, and Aaron M. Dollar, Senior Member, IEEE
}

\begin{abstract}
This paper presents a method for detecting contact by tracking a compliant structure's resonant frequency, which increases with external contact. The approach uses an inexpensive accelerometer mounted on or embedded inside the structure, and a phase locked loop circuit and actuator to oscillate the structure at its resonant frequency. This approach is applied to a robotic finger, and two contact detection metrics are compared. The first and best-performing metric detects changes in the frequency of oscillation. The second metric measures changes in the amplitude of the oscillation and detects contact based on amplitude attenuation. The frequency-based method was shown to be approximately three times as sensitive as the amplitude metric, capable of detecting contacts at between 0.03 and $1.65 \mathrm{~N}$, depending on the contact location, approach velocity, and finger stiffness, while the amplitude-based metric detected contacts at between 0.1 and $8 \mathrm{~N}$. The resonant frequency-based approach to contact detection resulted in reliable contact detection anywhere on the finger, including the finger pads, back of the finger, and even out of plane on the side of the finger.
\end{abstract}

Index Terms-Electromechanical sensors, manipulators, robot sensing systems, tactile sensors.

\section{INTRODUCTION}

$\mathbf{T}$ OUCH sensing is an important aspect of grasping and manipulation. Humans rely heavily on tactile sensing during reaching, grasping, and manipulation [1], and the tactile information utilized is diverse, ranging from force/pressure, skin stretch, vibration, slip, pressure, and temperature [1]-[3]. Naturally, many of these same modalities are useful or necessary for robotic grasping and manipulation. Research efforts to develop sensors for manipulation have spanned the range of both intrinsic/proprioceptive sensors and extrinsic/exteroceptive sensors [4]-[9].

In this paper, we expand upon our initial work [10] developing a contact sensor that is particularly useful for compliant articulated structures, including compliant hands such as the SDM Hand [11]. In general, the approach utilizes measurements of the frequency response of the digit in real time, with contact detection based upon a change in the resonant frequency. Unlike

Manuscript received February 15, 2013; revised July 18, 2013; accepted October 13, 2013. Date of publication November 14, 2013; date of current version May 16, 2014. Recommended by Technical Editor X. Tan. This work was supported in part by the National Science Foundation under Grant IIS-0953856 and in part by the Office of Naval Research under Grant N000141010737. A preliminary version of this paper was presented at the 2012 IEEE International Conference on Robotics and Automation, St. Paul, MN, USA, May 14-18.

The authors are with the Department of Mechanical Engineering and Materials Science, School of Engineering and Applied Science, Yale University, New Haven, CT 06511 USA (e-mail: spencer.backus@yale.edu; aaron.dollar@ yale.edu).

Digital Object Identifier 10.1109/TMECH.2013.2287761

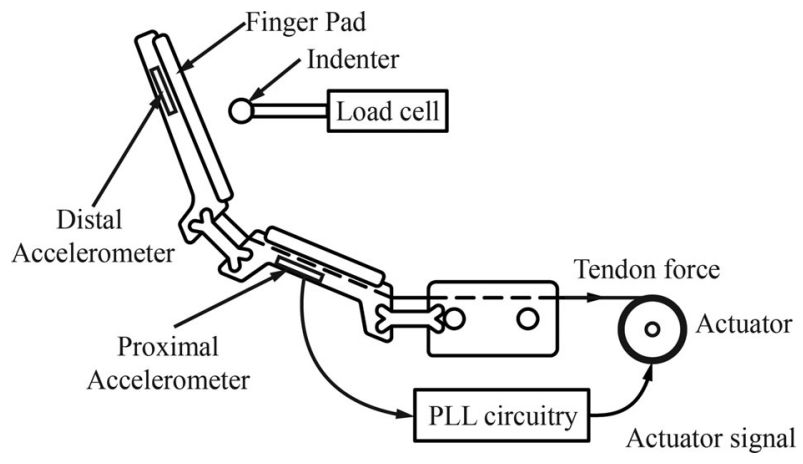

Fig. 1. Diagram of instrumented finger and test probe. The sensor system consists of an accelerometer connected to the PLL circuit. The output from the PLL modulates the current applied to the tendon actuator.

many traditional methods of sensing, which rely on expensive and oftentimes fragile custom-made sensors placed at the fingertips (or any surface where sensing is desired) [12], our approach uses a single, inexpensive accelerometer and a phase locked loop (PLL) integrated circuit to excite the system at its resonant frequency. By sensing changes in this resonant frequency, we are able to detect contact anywhere on the digit, not just on the instrumented surface as is typical in many other approaches.

Other researchers have investigated accelerometer and vibration-based contact sensors and have applied similar frequency characterization techniques, but have not combined these approaches in a frequency-based vibrational contact sensor. For example, Howe and Cutkosky [13] describe an accelerometer-based sensor designed to detect the onset of slip. This sensor consists of an accelerometer attached to a compliant skin wrapped around a rigid finger and detects the onset of slip by measuring the accelerations of the skin as it stretches and snaps back when it begins to slip. Although this approach reliably detects the onset of slip, the authors do not describe its performance as a contact sensor.

Motoo et al. [4] implemented an impedance-based contact sensor that consists of two piezoelectric elements separated by a polymer sheet. One of the piezoelectric elements is driven at a constant frequency while the other is used as a transducer to monitor the system's response. The signal from the piezoelectric element is used to measure the impedance of the polymer, and contact is detected when the measured mechanical impedance changes. Rudolf and Seemann [14] used a piezoelectric actuation scheme and a PLL to oscillate a beam at resonance, but did not investigate sensing.

Omata and Terunuma [5] have developed a related sensor for measuring the stiffness of soft materials such as biological tissues. This sensor consists of a load cell, piezoelectric driver, 
and piezoelectric transducer mounted on a probe that is applied to the tissue to be characterized. The system is excited at its resonant frequency by a circuit that tracks the resonant frequency of the probe and tissue system. The stiffness of the contacted tissue is then inferred based on the known stiffness of the probe, the compressive force applied to it, and the measured change in resonant frequency. Unlike our sensor, this sensor uses a load cell to characterize the contact and the change in resonant frequency to measure the stiffness of the material. Furthermore, it is designed to characterize contacts at the tip of the probe and is incapable of detecting contacts elsewhere on the probe. This sensor has been developed into a commercially available stand-alone instrument, the Axiom Biosensor (Fukushima-ken, Japan), and also a fingertip sensor capable of taking measurements at a single point on a manipulator finger [15].

In this paper, we present a contact detector that is composed of inexpensive accelerometers mounted within a compliant robotic finger (see Fig. 1) and a PLL circuit that drives the finger at its resonant frequency with the main finger actuator. Using the accelerometer, we can sense contact anywhere on the finger based on changes in the amplitude or frequency of the oscillation and compare various frequency and amplitude-based contact metrics.

We begin this paper with a description of our approach, utilizing a simple beam and elastic contact model to illustrate the concept. Next, we describe our experimental setup and test procedure, followed by an evaluation of the proposed concept in a wide range of contact conditions and locations on the finger. Finally, we discuss issues related to the practical implementation of the approach, including how the results presented here can be extended to additional contact conditions as well as to provide a richer information set about the contact state.

\section{APPROACH}

Our sensor operates by measuring the frequency response of the digit in real time and detects contact based upon observed changes in this response. For the test platform described in this paper, the finger consists of two rigid links connected by compliant joints. Although the actual nature of the contact is more complicated, we model the contact as a spring and damper in parallel with values equivalent to those of the finger pad material. (Note that the sensor does not require a model of the finger-it simply looks for changes in the baseline resonant frequency.)

In order to understand the basic resonant behavior of our finger and how it changes with external contacts, we present two simple models - a single-joint finger and a double-joint finger. First, as shown in the following single link and rotational joint example (see Fig. 2), if a contact is modeled as a spring and damper in parallel acting at a point on the link, the stiffness and damping of the contact add to the stiffness and damping of the joint and therefore change the overall frequency response of the system. As a result, the resonant frequency and oscillatory amplitude of the overall system changes as a function of both the location and stiffness of the contact as shown in (2). Because the change in frequency is a function of both the contact stiffness and
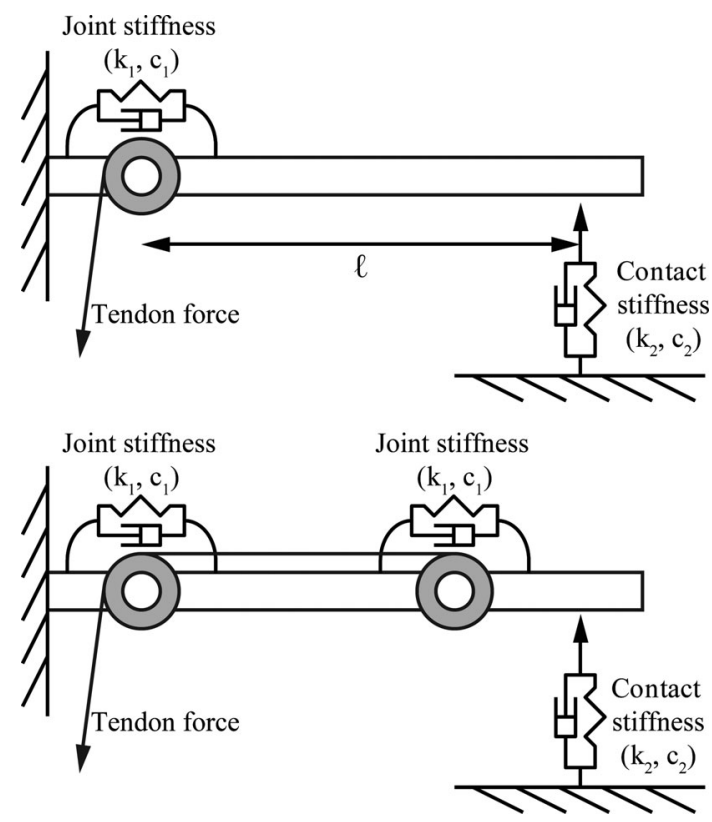

Fig. 2. Diagrams of simple single link and two link systems.

location, there is insufficient information to disambiguate which factor is responsible for the change in frequency and thereby determine the contact location or stiffness. Instead, the sensor functions by detecting this change in frequency or amplitude and thereby the underlying contact event, making it a binary contact detector in its current form. However, with a well-characterized finger, and known contact stiffness or location, it should be possible to predict the contact location or stiffness, respectively. However, this would require additional sensor information on the location or force applied by the contact.

The following two equations represent the angular acceleration of the rotational joint and the resonant frequency of the single link/contact system for small angles:

$$
\begin{aligned}
\ddot{\theta} & =\frac{1}{I}\left(-\left(k_{1}+k_{2} \ell_{1}^{2}\right) \theta-\left(c_{1}+c_{2} \ell_{1}^{2}\right) \dot{\theta}+u(t)\right) \\
\omega_{0} & =\frac{1}{2 \pi} \sqrt{\frac{k_{1}+k_{2} \ell_{1}^{2}}{I}} \mathrm{~Hz}
\end{aligned}
$$

where $I$ is the link's moment of inertia, $k_{1}$ and $c_{1}$ are the spring and damping constants of the joint, $k_{2}, c_{2}$, and $l_{1}$ are the contact spring and damping constants and the contact location, and $u(t)$ is the excitation input. As shown in (2), the resonant frequency is only a function of the digits moment of inertia, the joint's stiffness, and the contact's location and stiffness, assuming the object is fixed in space. When contact occurs, the contact stiffness, $k_{2}$, increases from zero to the combined stiffness of the finger pad and object, causing the resonant frequency to increase.

The full two link system was modeled using Simulink and linearized about its equilibrium. The frequency response of the linearized model was then analyzed using Matlab. The results from this analysis are presented in the frequency response plot shown in Fig. 3. As can be seen from the locations of the peaks in this plot, the system exhibits two resonant frequencies, a primary 
Frequency Response

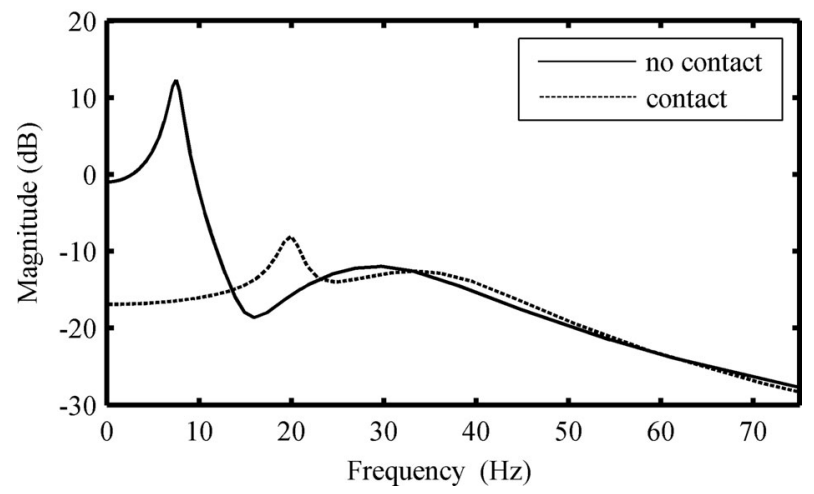

Fig. 3. Predicted frequency response of the two link system without contact (solid line) and with $100 \mathrm{~N} / \mathrm{m}$ contact (dashed line).

one at approximately $10 \mathrm{~Hz}$ and a substantially weaker mode at approximately $25 \mathrm{~Hz}$. In practice, these two modes correspond to the frequencies observed in response to a step input and the resonant frequency tracked by the PLL, respectively. When contact is included, the frequency of both modes increases to approximately 20 and $40 \mathrm{~Hz}$, respectively. Both the predicted resonant frequencies and the increase in resonant frequency predicted by the model correspond to the observed behavior of the system. However, because of some simplifications made in the ideal model such as ignoring the coulomb damping caused by the friction between the tendon and tendon sheath, and the out of plane vibratory modes of the joints, the actual frequency response of the system with and without contact is somewhat more complicated than our model predicts.

\section{METHODS}

\section{A. Experimental Setup}

We have implemented this contact sensor on a two link polymer flexure-based finger derived from the SDM Hand design [11]. The digit, shown in Figs. 1 and 3, is actuated by a current-controlled de motor (Maxon 2140.937-58.236-050) via a single distally terminated tendon. This actuator applies both the force to flex the digit as well as the oscillatory force used to excite the digit at its resonant frequency. The digit is instrumented with two three-axis accelerometers (model adx1335, Texas Instruments) one embedded in the proximal link and the other in the distal link of the finger. Currently, only the $z$-axis channel is monitored and it is oriented normal to the finger pad in each link. Although two accelerometers are included, the work shown in this paper only considers one or the other at a time.

Tracking of the resonant frequency in the physical system is achieved using a PLL. The system first measures the oscillation of the proximal link of the digit normal to the finger pad with the accelerometer. Then, the accelerometer signal is filtered and digitized to meet the requirements of the PLL integrated circuit (model cd4046be, Texas Instruments). The dc bias is removed from the signal by a second-order high-pass filter. This filter is implemented using a UA741 op-amp and has a cutoff frequency of $4.8 \mathrm{~Hz}$. The signal is digitized using an op-amp comparator implemented with an LM324 op-amp. The digital signal is then passed to the IC-based PLL, which generates the periodic excitation signal that specifies the current driving the tendon actuator. The PLL tracks the resonant frequency of the system by performing feedback control on the phase difference between the oscillation of the finger and the periodic excitation signal that it generates. The excitation signal generated by the PLL in turn specifies the current supplied to the motor and the excitation force applied to the digit. Therefore, the PLL is controlling the phase difference between the force applied to the finger and its response, thereby exciting it at resonance. The amplitude of the driving signal was adjusted so that the distal joint oscillates through approximately $0.9^{\circ}$ when not in contact with an object. This small amplitude oscillation is sufficient to generate a clear signal from the accelerometer but minimizes the displacement of the finger (and is barely noticeable by sight).

\section{B. Contact Criteria}

Two frequency-based metrics and two amplitude-based metrics were tested. They are the following:

1) increase in frequency measured by the proximal accelerometer;

2) increase in frequency measured by the distal accelerometer;

3) decrease in amplitude measured by the proximal accelerometer;

4) decrease in amplitude measured by the distal accelerometer.

The criteria for contact detection for all of these metrics are based upon the comparison of an initial baseline sample of the parameter (e.g., the frequency of oscillation measured by the accelerometer) collected during system initialization when the finger is not in contact to the real-time measurement of the parameter, both of which are filtered using a ten sample moving average (the last second of data in the current implementation). When powered on, the system is given $60 \mathrm{~s}$ to reach equilibrium, after which the mean frequency and the amplitude of a 1-s sample are measured. The threshold level above which contact is indicated is then set based on these baseline measurements. Adjusting this threshold is a tradeoff between detector sensitivity and accuracy: a lower threshold above the initial mean frequency results in greater sensitivity to extremely light contacts but also introduces a larger number of false positives. For the experiments presented in this paper, the frequency threshold is set as three standard deviations above the measured mean frequency. Similarly, the amplitude threshold is set at $90 \%$ of the measured mean amplitude of the accelerometer signal. Contact is detected when the real-time measurement crosses these preset thresholds.

\section{Test Procedure}

The contact system was evaluated based upon its ability to detect both initial contact events and its static contact state. The test setup, shown in Fig. 4, consists of the finger/sensor/actuator system affixed to the bench top and a load cell (model MDB5 , Transducer Techniques, $0-5 \mathrm{lbf}$ range), terminating in a 


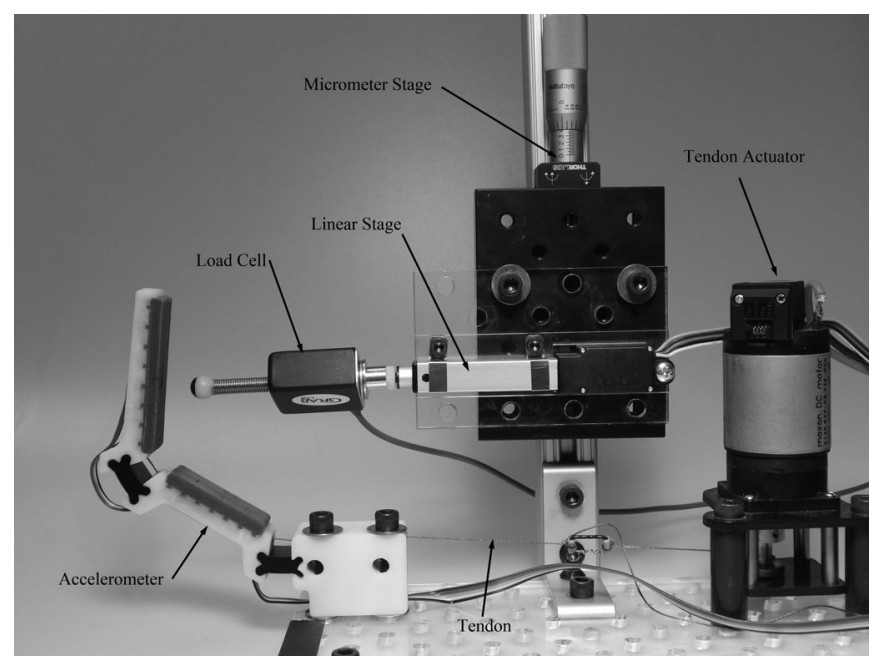

Fig. 4. Instrumented finger and test probe.

$6.35 \mathrm{~mm}\left(0.25^{\prime \prime}\right)$ diameter spherical nylon tip through which contact is made with the finger. The load cell and nylon probe are mounted on a voltage-controlled linear actuator (Firgelli, L12-50-50-6-P) that is used to advance the probe at constant velocity until it contacts the digit. The entire liner probe stage is mounted on a micrometer stage to adjust the contact location on the finger. For each area, the translation axis of the micrometer stage was manually aligned with the surface of the finger so that the probe was normal to the contact surface.

The transient behavior of the system was measured by advancing the probe at a constant velocity until contact was detected. The sensor's performance was compared to direct contact detection based upon contact force measurements taken at the same time using the load cell in series with the probe. Performance was quantified in terms of the elapsed time after contact was first measured by the load cell as well as measured contact force at the point when the sensor detected contact. This test was repeated for a range of velocities from 8 to $30 \mathrm{~mm} / \mathrm{s}$ and over a range of contact locations on both the compliant finger pads and back of both the proximal and distal links. Out of plane contacts, where the probe contacted the side of the finger, were also evaluated. The stiffness of the contact was also varied by wrapping the indenter in foams of varying stiffness. Repeatability of the sensor was also tested by repeating each trial 24 times and computing the mean and standard deviation of each set of trials.

\section{RESULTS}

In operation, the system exhibits three different oscillatory behaviors depending on the contact state, examples of which are shown in Fig. 5. Prior to contact, the digit exhibits a clearly periodic oscillation with a dominant frequency of approximately $29.6 \mathrm{~Hz}$, shown in Fig. 5(a). At low contact force (on the order of $0.1 \mathrm{~N}$ ), a transitional phase occurs in which the link bounces off of the contact (i.e., has a "flight" phase) and the resonant frequency rises slightly to around $30.8 \mathrm{~Hz}$ (as a result of averaging the force between the contact and flight phases) and the
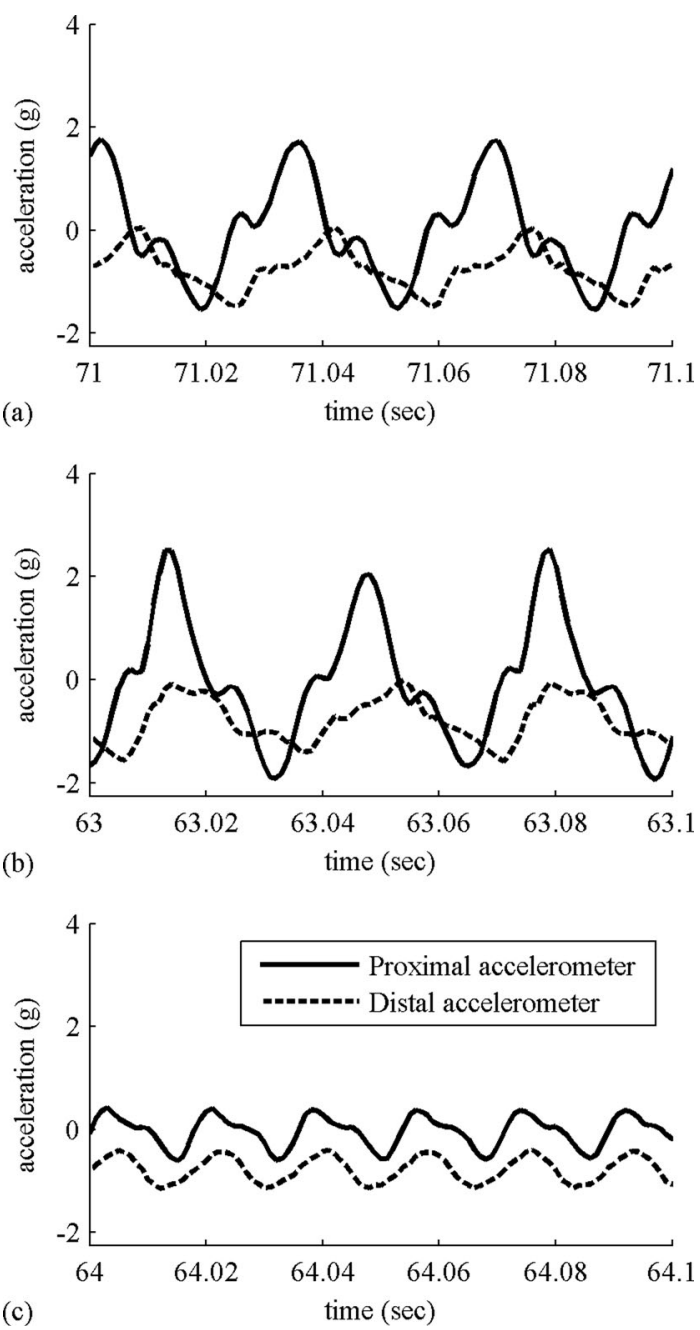

Fig. 5. Measured acceleration of the digit under no (a) contact, (b) light contact, and (c) full contact conditions. Contact for all three cases is made in the center of the distal finger pad.

peak amplitude of the accelerometer signal increases as shown in Fig. 5(b). Finally, if the contact force is increased, the bouncing/flight phase disappears, the resonant frequency rises, and its amplitude decreases substantially as shown in Fig. 5(c). In this case, the load cell measured an average contact force of approximately $0.4 \mathrm{~N}$ and the resonant frequency rises to $57 \mathrm{~Hz}$.

These changes can also be analyzed in the frequency domain as shown in the power spectral density plot of the signal shown in Fig. 6. Here, we can see that the dominant vibratory mode in the noncontact case is at $29.8 \mathrm{~Hz}$. This rises to $30.8 \mathrm{~Hz}$ once contact is made. Finally, when the contact force is increased, the vibratory modes around $30 \mathrm{~Hz}$ are attenuated and the dominant resonant mode moves to $57.1 \mathrm{~Hz}$. Higher harmonic are also present and exhibit a similar change when contact is made: for example, the first large harmonic at $43.9 \mathrm{~Hz}$ rises to $45.9 \mathrm{~Hz}$ when contact is made and is attenuated when the contact force is increased.

Figs. 7 and 8 show the response of the frequency- and amplitude-based metrics to a representative contact event. In this trial, the probe was advanced at $7.6 \mathrm{~mm} / \mathrm{s}$ and made contact 


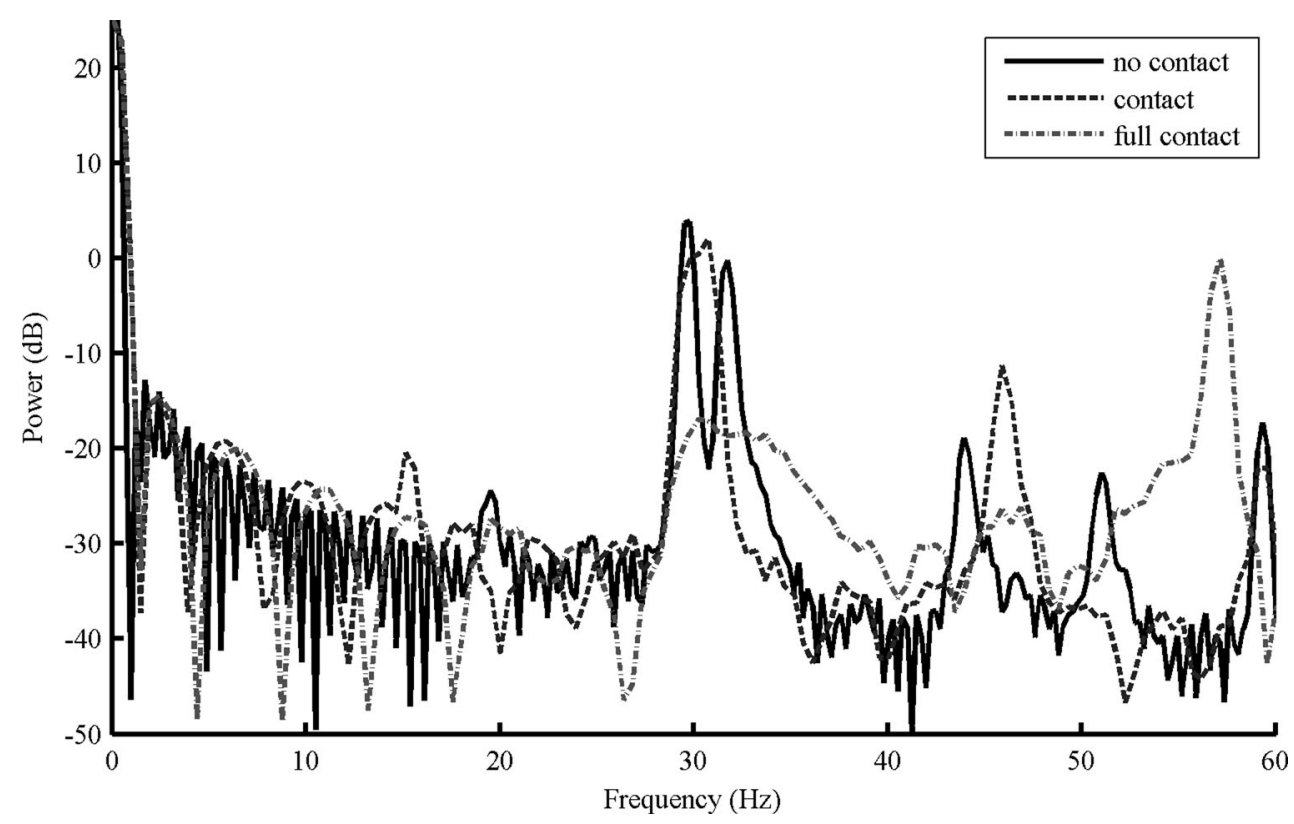

Fig. 6. Power spectral density of the distal accelerometer signal with and without contact.

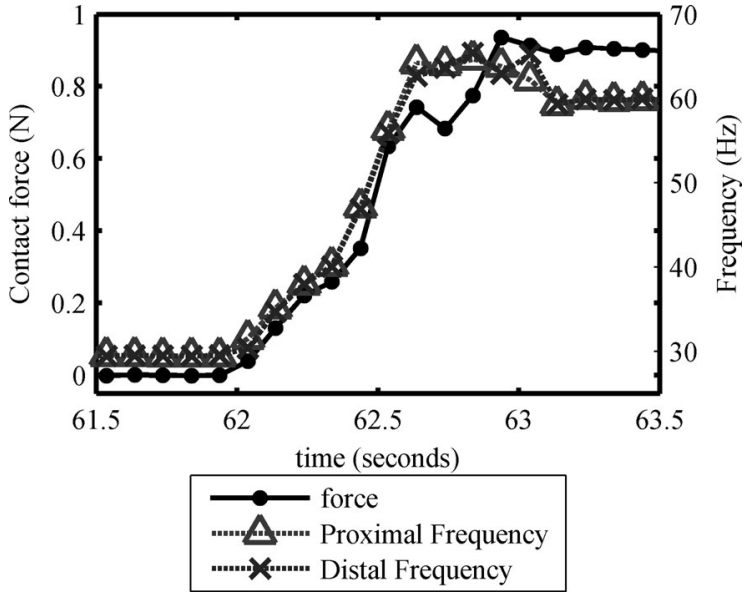

Fig. 7. Measured force and proximal, and distal accelerometer frequencies during a typical contact event.

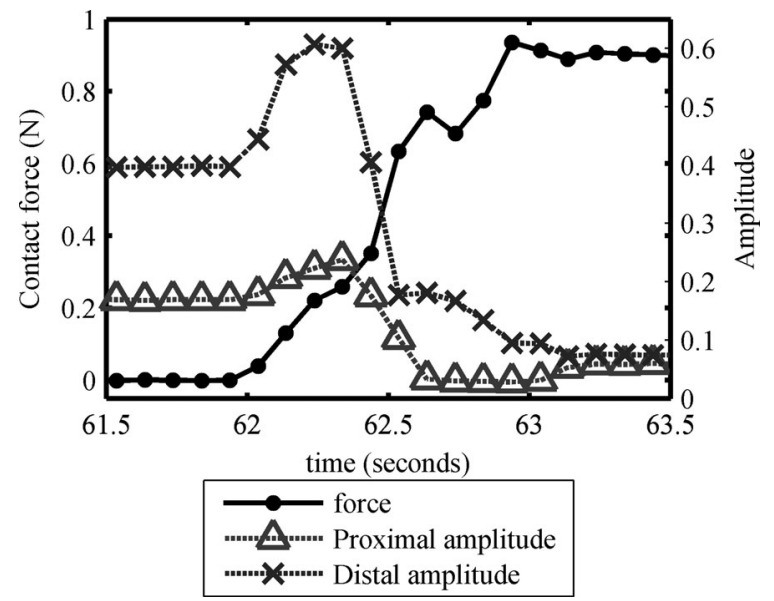

Fig. 8. Measured force and proximal and distal amplitude change during a typical contact event.

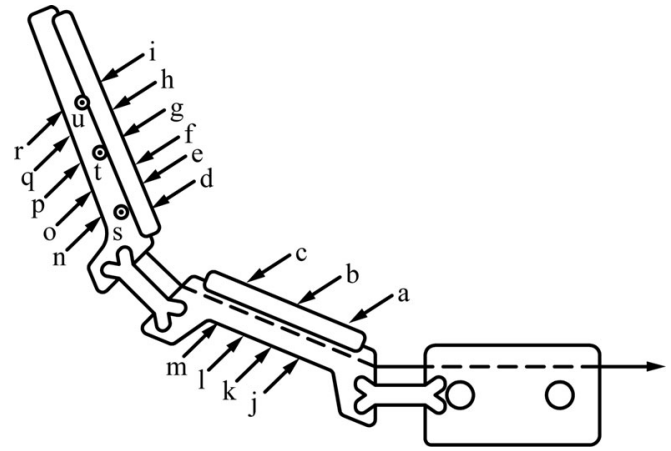

Fig. 9. Contact test locations.

on the proximal end of the distal finger pad (location $d$ ). For the frequency-based metric, Fig. 7, contact is registered by the load cell at $t=62.04 \mathrm{~s}$ when the probe force rises from 0 to $0.039 \mathrm{~N}$. Similarly, contact is detected at $t=62.04 \mathrm{~s}$ when the frequency rises from 29.45 to 31.5 and $30.37 \mathrm{~Hz}$ for the proximal and distal accelerometers, respectively.

Fig. 8 shows the results for the amplitude-based metric. When contact is initially made at $t=62.04 \mathrm{~s}$, both the proximal and distal amplitudes rises before both drop below their thresholds and contact is registered at $t=62.54 \mathrm{~s}$ by both the proximal and distal amplitude metrics. This rise is due to the finger bouncing off of the contact during the partial contact phase, causing large accelerations.

The aggregated results of a set of similar trials at various locations on the finger for each contact metric are presented in Table I and the contact locations are shown in Fig. 9. Figs. 10 and 11 show examples of the relationship between the contact location, velocity, and stiffness, and the force threshold and time delay for each contact metric. In Figs. 10(a) and 11(a), the contact velocity is held constant at $8 \mathrm{~mm} / \mathrm{s}$ and the contact location is varied over a $1^{\prime \prime}$ region on the distal finger pad. 
TABLE I

SUMMARY OF TRANSIENT CONTACT TEST DATA

\begin{tabular}{|c|c|c|c|c|c|c|c|c|c|c|c|}
\hline \multirow{2}{*}{$\begin{array}{l}\text { contact } \\
\text { location }\end{array}$} & & \multirow{2}{*}{$\begin{array}{l}\text { Distal from } \\
\text { joint }(\mathrm{mm})\end{array}$} & \multirow{2}{*}{$\begin{array}{c}\text { speed } \\
(\mathrm{mm} / \mathrm{sec})\end{array}$} & \multicolumn{2}{|c|}{ Proximal frequency } & \multicolumn{2}{|c|}{ Distal frequency } & \multicolumn{2}{|c|}{ Proximal amplitude } & \multicolumn{2}{|c|}{ Distal amplitude } \\
\hline & & & & $\begin{array}{l}\text { Time } \\
\text { (sec) }\end{array}$ & $\begin{array}{c}\text { force at } \\
\text { contact }(N)\end{array}$ & $\begin{array}{l}\text { Time } \\
\text { (sec) }\end{array}$ & $\begin{array}{c}\text { force at } \\
\text { contact }(N)\end{array}$ & $\begin{array}{l}\text { Time } \\
\text { (sec) }\end{array}$ & $\begin{array}{c}\text { force at } \\
\text { contact }(N)\end{array}$ & Time (sec) & $\begin{array}{c}\text { force at } \\
\text { contact }(N)\end{array}$ \\
\hline \multirow[t]{2}{*}{ a } & mean & 11.6 & 6.82 & 0.2472 & 1.6494 & 0.1427 & 0.4254 & 4.0408 & 8.2773 & - & - \\
\hline & $\sigma$ & & & 0.0608 & 0.2573 & 0.0504 & 0.0834 & 1.8543 & 2.5533 & - & - \\
\hline \multirow[t]{2}{*}{$\mathrm{b}$} & mean & 24.3 & 9.16 & 0.0703 & 0.3270 & 0.0841 & 0.3963 & 0.9155 & 2.4163 & - & - \\
\hline & $\sigma$ & & & 0.0598 & 0.1554 & 0.0528 & 0.1865 & 0.1094 & 0.2812 & - & - \\
\hline \multirow[t]{2}{*}{ c } & mean & 37.0 & 8.76 & 0.1432 & 0.3426 & 0.0427 & 0.4254 & 0.5423 & 0.8961 & - & - \\
\hline & $\sigma$ & & & 0.0554 & 0.0902 & 0.0504 & 0.0834 & 0.0572 & 0.0618 & - & - \\
\hline \multirow[t]{2}{*}{$\mathrm{d}$} & mean & 14.6 & 8.20 & 0.15 & 0.1404 & 0.0883 & 0.0822 & 0.7158 & 0.6339 & 0.8279 & 0.8337 \\
\hline & $\sigma$ & & & 0.0517 & 0.0390 & 0.0618 & 0.0277 & 0.0391 & 0.0383 & 0.0474 & 0.0858 \\
\hline \multirow[t]{2}{*}{ e } & mean & 19.68 & 8.36 & 0.0713 & 0.0919 & 0.1048 & 0.1143 & 0.5529 & 0.4960 & 0.6512 & 0.4911 \\
\hline & $\sigma$ & & & 0.0475 & 0.0264 & 0.0446 & 0.0295 & 0.0696 & 0.0255 & 0.0650 & 0.0352 \\
\hline \multirow[t]{2}{*}{ f } & mean & 24.76 & 8.34 & 0.1782 & 0.0910 & 0.1600 & 0.0959 & 0.7387 & 0.4853 & 0.8387 & 0.5679 \\
\hline & $\sigma$ & & & 0.0388 & 0.0146 & 0.1329 & 0.0273 & 0.0483 & 0.1326 & 0.0601 & 0.1735 \\
\hline \multirow[t]{2}{*}{ g } & mean & 29.84 & 7.92 & 0.0198 & 0.0346 & 0.0675 & 0.0485 & 0.4017 & 0.1038 & 0.5234 & 0.2804 \\
\hline & $\sigma$ & & & 0.0459 & 0.0165 & 0.0599 & 0.0176 & 0.0705 & 0.0318 & 0.0529 & 0.0696 \\
\hline \multirow[t]{2}{*}{$\mathrm{g}$} & mean & 29.84 & 19.68 & 0.0513 & 0.0653 & 0.0926 & 0.0946 & 0.2573 & 0.5265 & 0.2732 & 0.5043 \\
\hline & $\sigma$ & & & 0.0618 & 0.0296 & 0.0415 & 0.0314 & 0.0686 & 0.0536 & 0.0772 & 0.0747 \\
\hline \multirow[t]{2}{*}{$\mathrm{g}$} & mean & 29.84 & 29.74 & 0.0392 & 0.1740 & 0.0283 & 0.1199 & 0.2652 & 0.5421 & 0.2768 & 0.5568 \\
\hline & $\sigma$ & & & 0.0773 & 0.0949 & 0.0606 & 0.0753 & 0.1534 & 0.1527 & 0.1676 & 0.1651 \\
\hline \multirow[t]{2}{*}{$\mathrm{h}$} & mean & 34.92 & 9.31 & 0.1442 & 0.0456 & 0.1862 & 0.0547 & 0.5457 & 0.1693 & 0.5086 & 0.2929 \\
\hline & $\sigma$ & & & 0.0774 & 0.0156 & 0.0625 & 0.0096 & 0.2757 & 0.1350 & 0.1536 & 0.1080 \\
\hline \multirow[t]{2}{*}{$\mathrm{i}$} & mean & 40 & 7.90 & 0.1286 & 0.0291 & 0.1280 & 0.0339 & 0.4871 & 0.1003 & 0.6022 & 0.1869 \\
\hline & $\sigma$ & & & 0.0734 & 0.0084 & 0.0562 & 0.0078 & 0.0914 & 0.0651 & 0.0846 & 0.0784 \\
\hline \multirow[t]{2}{*}{$\mathrm{j}$} & mean & 21.95 & 6.45 & 0.0862 & 0.3718 & 0.0672 & 0.6144 & 2.5212 & 4.4012 & 5.0693 & 4.2245 \\
\hline & $\sigma$ & & & 0.1517 & 0.2314 & 0.0486 & 0.2656 & 0.0772 & 0.1058 & 2.7336 & 0.0838 \\
\hline $\mathrm{k}$ & mean & 28.3 & 7.97 & 0.0469 & 0.3346 & 0.0816 & 0.4997 & 0.3353 & 1.0614 & 3.1095 & 3.6190 \\
\hline & $\sigma$ & & & 0.0691 & 0.2117 & 0.0687 & 0.1968 & 0.0615 & 0.2993 & 0.1483 & 0.0887 \\
\hline 1 & mean & 34.65 & 8.75 & 0.0628 & 0.3618 & 0.0667 & 0.3145 & 0.3033 & 0.7147 & 1.0547 & 1.9824 \\
\hline & $\sigma$ & & & 0.0497 & 0.1496 & 0.1050 & 0.1614 & 0.1869 & 0.1622 & 0.0591 & 0.0906 \\
\hline $\mathrm{m}$ & mean & 41.0 & 8.44 & 0.0804 & 0.3219 & 0.0916 & 0.3690 & 0.2930 & 0.6094 & 0.8496 & 1.4252 \\
\hline & $\sigma$ & & & 0.0374 & 0.1206 & 0.0283 & 0.1273 & 0.0680 & 0.1911 & 0.1580 & 0.1255 \\
\hline $\mathrm{n}$ & mean & 22.6 & 8.03 & 0.0481 & 0.0789 & 0.0769 & 0.1001 & 0.3990 & 0.9115 & 0.5052 & 1.0620 \\
\hline & $\sigma$ & & & 0.0539 & 0.0374 & 0.0703 & 0.0275 & 0.0529 & 0.2310 & 0.0886 & 0.2117 \\
\hline o & mean & 28.5 & 8.05 & 0.1065 & 0.1128 & 0.1704 & 0.2392 & 0.3699 & 0.6975 & 0.4507 & 0.8528 \\
\hline & $\sigma$ & & & 0.0573 & 0.0953 & 0.0768 & 0.1220 & 0.1260 & 0.1899 & 0.1274 & 0.1644 \\
\hline $\mathrm{p}$ & mean & 35.3 & 8.62 & 0.1070 & 0.0912 & 0.0808 & 0.0871 & 0.3054 & 0.5231 & 0.3346 & 0.5371 \\
\hline & $\sigma$ & & & 0.0625 & 0.0999 & 0.0482 & 0.0575 & 0.1130 & 0.1457 & 0.1071 & 0.1589 \\
\hline$q$ & mean & 41.65 & 7.91 & 0.0718 & 0.0673 & 0.1000 & 0.0915 & 0.2387 & 0.2493 & 0.2265 & 0.2648 \\
\hline & $\sigma$ & & & 0.0453 & 0.0434 & 0.0508 & 0.0627 & 0.0977 & 0.1409 & 0.0683 & 0.1310 \\
\hline $\mathrm{r}$ & mean & 48.0 & 8.08 & 0.0538 & 0.0604 & 0.1972 & 0.0761 & 0.1353 & 0.1785 & 0.1576 & 0.2057 \\
\hline & $\sigma$ & & & 0.0506 & 0.0479 & 0.0998 & 0.0570 & 0.0788 & 0.1277 & 0.0593 & 0.0781 \\
\hline $\mathrm{s}$ & mean & 22.6 & 8.96 & 0.5162 & 0.7637 & 0.5888 & 0.8772 & 0.4860 & 0.7279 & 1.7309 & 2.0569 \\
\hline & $\sigma$ & & & 0.0664 & 0.0940 & 0.0938 & 0.1206 & 0.0771 & 0.0800 & 0.1237 & 0.1271 \\
\hline $\mathrm{t}$ & mean & 35.3 & 8.98 & 0.3421 & 0.4018 & 0.3895 & 0.3910 & 0.6765 & 0.7172 & 1.3925 & 1.2428 \\
\hline & $\sigma$ & & & 0.0598 & 0.0463 & 0.0677 & 0.0363 & 0.1527 & 0.1042 & 0.1863 & 0.1223 \\
\hline $\mathrm{u}$ & mean & 48.0 & 8.73 & 0.4890 & 0.3574 & 0.4600 & 0.3385 & 0.5588 & 0.3974 & 1.0738 & 0.7092 \\
\hline & $\sigma$ & & & 0.0571 & 0.0324 & 0.0507 & 0.0221 & 0.0508 & 0.0221 & 0.0756 & 0.0246 \\
\hline
\end{tabular}

Figs. 10(b) and 11(b) show the effect of the contact velocity when the contact location and stiffness are held constant. Finally, Figs. 10(c) and 11(c) show the effect that the stiffness of the contact has on the contact metric's sensitivity.

As shown in Fig. 10(a), the contact force threshold increases for more proximal contacts and ranges from $\sim 0.03$ to $\sim 0.14 \mathrm{~N}$ for the proximal frequency metric, $\sim 0.03$ to $\sim 0.1 \mathrm{~N}$ for the distal frequency metric, $\sim 0.1$ to $\sim 0.64 \mathrm{~N}$ for the proximal amplitude metric, and from $\sim 0.2$ to $0.8 \mathrm{~N}$ for the distal amplitude metric. These results compare favorably with direct contact detection using the load cell which detected contact at between $\sim 0.014$ and $\sim 0.19 \mathrm{~N}$.

The time delay between when contact was detected by the load cell and by the frequency-based contact metrics did not vary appreciably with location, as shown in Fig. 11(a). For these metrics, the delay varied from 0.00 to $0.30 \mathrm{~s}$ between all trials and, on average, there was a $\sim 0.12$-s delay. However, the time delay for the amplitude-based metrics did vary with contact location, increasing from 0.6 to $0.8 \mathrm{~s}$ for more proximal contacts.

In addition to these results for contacts on the distal finger pad, additional tests were performed at multiple locations on the proximal finger pad, back of both the proximal and distal links, and the side of the distal link. The results from these trials are summarized in Table I. Similar trends to the ones discussed for the contacts on the distal finger pad are present for contacts made on the other parts of the finger that were tested. However, the range of the minimum and maximum contact force and time delay for contacts on a given part of the finger varies significantly as a function of the link on which the contact is made and the contact surface. For example, the proximal frequency metric ranges from 0.34 to $1.6 \mathrm{~N}$ for contacts made on the proximal finger pad and from 0.35 to $0.76 \mathrm{~N}$ for contacts made to the side of the distal link. This variation in sensitivity as a function of 


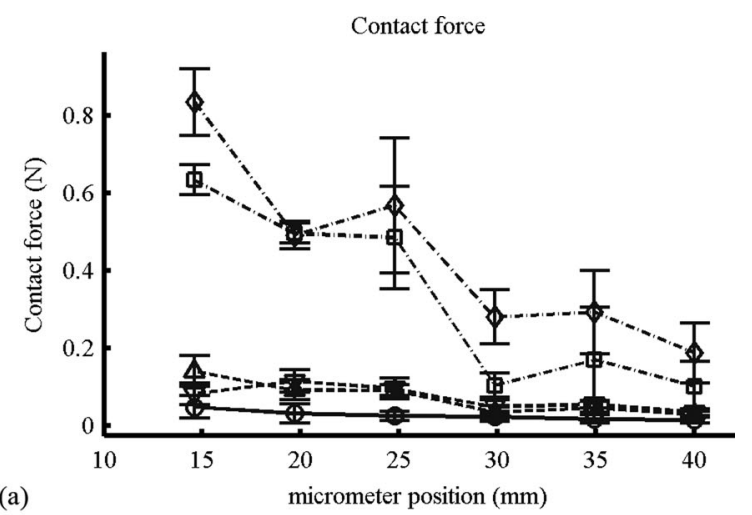

(a)
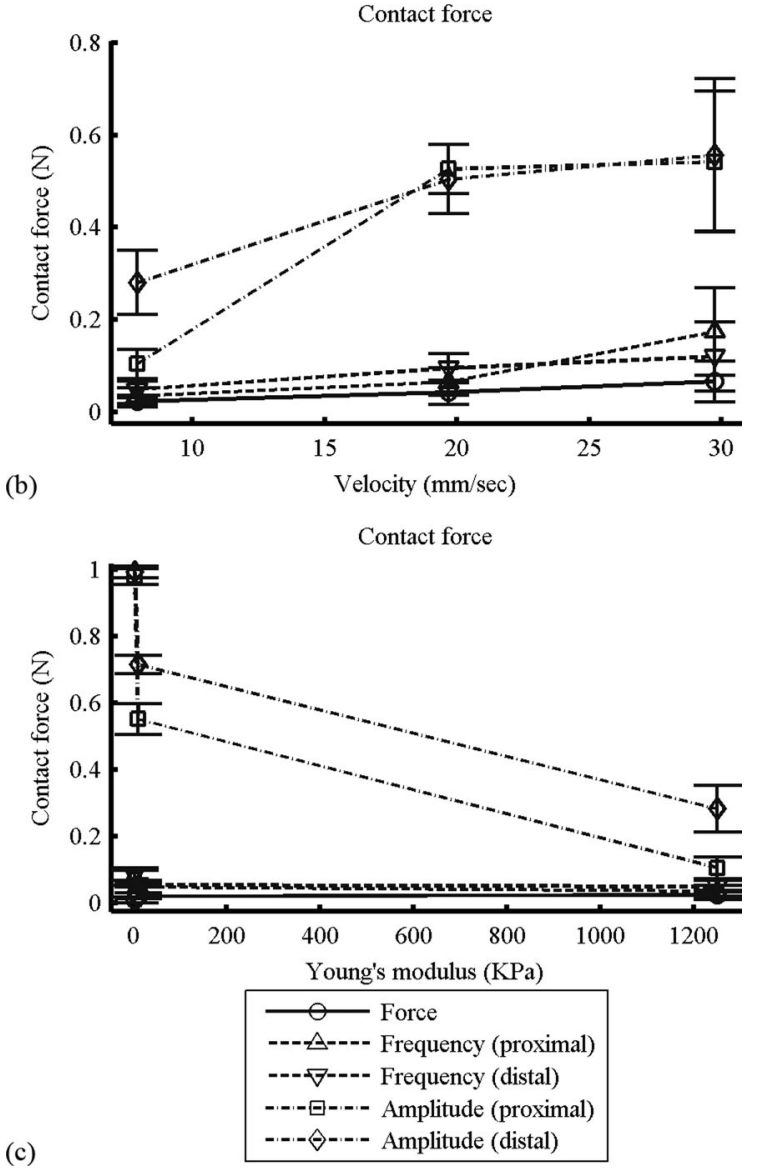

Fig. 10. Effect of (a) contact location, (b) velocity, and (c) stiffness on the contact force.

contact location is not surprising due to the differing moment arm of the contacts for contacts on the proximal link and different material stiffness and mechanical interaction for contacts on the side of the finger. Also, the distal amplitude metric fails to detect contact on the proximal link entirely.

Contact velocity also has an effect on the force threshold and time delay of the contact metrics as shown in Figs. 10(b) and 11(b) and Table I. Fig. 10(b) shows how increasing the contact velocity increases the contact force threshold of all of the contact metrics. Fig. 11(b) shows how increasing the speed decreases the time delay for the amplitude-based contact metrics but does not significantly change the time delay for the frequency-based
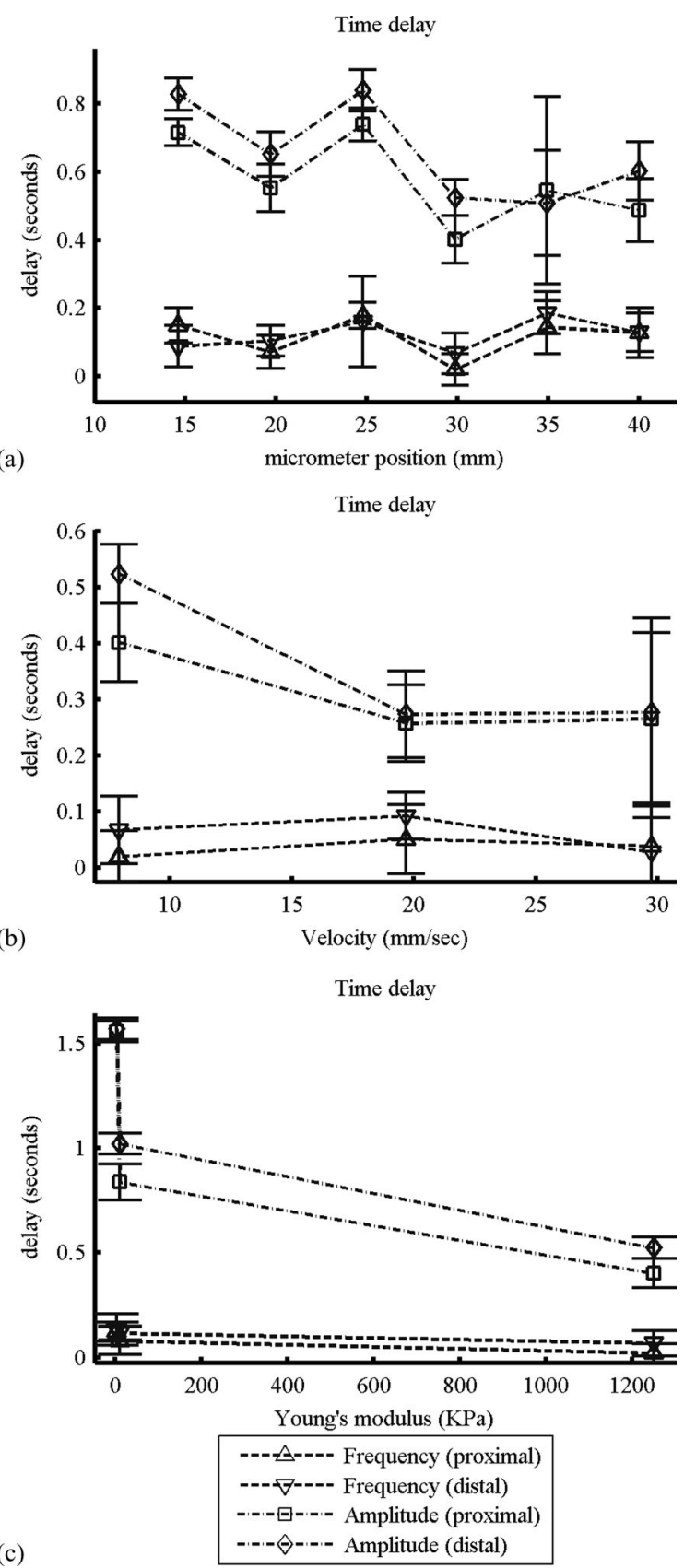

Fig. 11. Effect of (a) contact location, (b) velocity, and (c) stiffness on the time delay between the onset of contact and when it is registered by the sensor.

metrics. Figs. 10(c) and 11(c) show the effect the contact stiffness has on the force threshold and time delay. The contact stiffness clearly affects the force threshold and time delay of the amplitude-based metrics but does not appear to impact the frequency-based metrics. Finally, it is worth noting that the sensor also reliably detects the loss of contact and is insensitive to changes in the orientation of the finger.

\section{DISCUSSION}

As shown in Figs. 5 and 6, the accelerometer signals and their frequency spectrum under the no-contact and contact states are visibly distinguishable. As the probe is advanced past the point 
where contact is initiated, the oscillatory frequency continues to increase, while the amplitude first increases before decreasing. Although in this instance the changes in frequency and amplitude correspond to the increase in contact force, other factors including the contact stiffness, location, and flexion of the finger also affect the amplitude and the frequency of the finger. Therefore, the substantial frequency change that occurs as the contact force increases cannot be used as a quantitative measure of contact force. However, this information combined with additional sensory knowledge from an additional sensor (e.g., contact location on finger), could be combined to provide useful information about the contact force or stiffness.

Although the system's ability to distinguish between static contact and noncontact states is useful, its ability to accurately detect the onset or loss of contact is also important in many applications. As such, we evaluated the performance of each contact metric in terms of both the force threshold at which contact was sensed using the accelerometer and time delay between the onset (as sensed by the force transducer) and detection of contact over a range of contact locations, velocities, and stiffnesses. These tests show that in general the frequency-based metrics perform significantly better than the amplitude-based metrics, as can be seen by comparing the average time delay and force threshold for each metric in Table I and by the greater time delay and force at different contact locations, velocities, and stiffnesses in Figs. 10 and 11. These tests also show that, in general, the sensor detects more proximal contacts at a higher force threshold and longer time delay than distal contacts.

The longer time delay and higher force threshold exhibited by the amplitude-based metrics can be explained by the system's behavior during contact shown in Figs. 7 and 8. As shown in Fig. 7, the resonant frequency of the digit rises above the frequency threshold shortly after contact is made. In contrast, as shown in Fig. 8, the amplitude of the signal initially rises when contact is made at a low force. This is caused by the digit entering a bouncing contact regime at low contact forces. Only after the contact force increases further does the amplitude fall below the noncontact amplitude and register as a contact using the amplitude-based contact metric.

In addition to the contact metric used to detect contact, the location, velocity, and stiffness all can affect the force threshold of the sensor. As shown in Fig. 10(a), contacts made more proximally on the finger are detected at a higher force threshold. This can be explained by the force and moment arm at which the contact is made: more distal contacts can exert the same moment with a lower contact force than more proximal contacts due to the longer moment arm. This relationship holds for all locations tested on both the proximal and distal link, as shown in the data for these tests in Table I. Although unavoidable, this relationship is undesirable in applications where a specific maximum contact force is critical. In the worst case, where a contact is made on the proximal end of the proximal link of the finger (location $a$ ), the contact force threshold is over ten times higher than for a contact on the tip of the finger, raising the effective force threshold of the sensor substantially.

The small slopes in Fig. 11(a) show that the location has less of an effect on the time delay for the sensor detecting contacts on similar surfaces. There is a statistically significant relationship between the contact location and time delay for the amplitude-based metrics but not for the frequency-based metrics. This variation in time delay can also be explained by the force/moment arm relationship that causes the variation in force threshold: the probe has to advance further, resulting in a longer delay for the more proximal higher contact forces. Although the variation in time delay as a function of position is also undesirable, the much less pronounced dependence for the amplitude-based metrics and lack of detectable dependence for the frequency-based metrics means that one can assume a reasonably constant delay between onset and detection of contact, regardless of the position for similar contact events. Although this trend is present for contacts at all locations on the digit, the magnitude of the delay varies significantly for contacts made on different surfaces of the digit as shown in the data presented in Table I.

Like location, contact velocity also has a somewhat linear relationship with contact force at contact as shown in Fig. 10(b) and a relationship with the time delay for the amplitude but not frequency-based metrics as shown in Fig. 11(b). Although not captured by our theoretical model that did not consider the transient behavior of the PLL, the relationship between velocity and force can be explained by the fact that there is a slight delay between the onset and detection of contact, and during this delay the probe will advance further when it is driven at a higher velocity, resulting in a higher contact force. Similarly, at lower velocities it takes longer before the probe advances far enough to register as a contact, resulting in a longer time delay. This relationship for the amplitude-based metrics has the beneficial result that higher velocity contacts are detected more quickly, allowing the system to react faster when contact velocities and forces are higher.

Finally, contact stiffness, especially for extremely low stiffnesses plays a significant role in the force threshold and time delay of the system. As shown in Figs. 10(c) and 11(c), stiffer contacts result in lower contact forces and time delays, as predicted by our model of the system. Altering the stiffness of the probe contacting the finger changes the overall contact stiffness: adding a softer material will result in a decrease in the resonant frequency and therefore can be detected more slowly. Although an ideal sensor would not exhibit any dependence on the stiffness of the object, this behavior has the desirable benefit that the system responds faster and at lower contact forces for hard contacts where damaging the gripper or object is most likely.

Although it is difficult to precisely quantify differences between various contact sensors due to their different operating modalities and implementations, we will attempt to compare the performance and limitations of our system to sensors used in a number of other research hands. Most robotic hands rely on intrinsic sensors that measure the forces across joints or extrinsic sensors such as resistive or capacitive force sensors on the finger pad. Examples of intrinsic sensors include the miniature six-axis load cells used in the DLR II hand or the strain gaugebased tendon tension sensors used in the Utah/MIT Hand. These sensors measure the load within a link or about a joint, respectively. Their resolution depends heavily on the design of the load 
cell and associated signal conditioning electronics, and in the case of the DLR Hand, the load cell has a resolution of between 0.005 and $0.02 \mathrm{~N}$ depending on the measurement axis [9]. Similarly, their ability to measure contacts depends on being able to measure a force in the direction of the contact, limiting sensors such as the tendon tension sensor to only measuring contacts that exert a moment about the joint axis [16].

Common extrinsic sensors include force sensitive resistors (FSR) or capacitive sheets placed on or under the finger pad. One commercial example of this technology is the finger pad contact sensor array used in the Barrett Hand, which has a maximum resolution of $0.01 \mathrm{~N}$ per sensing element [17]. Other researchers have attempted to use FSR's as a low cost alternative to purpose designed contact sensors but have had difficulty placing the sensors on irregular surfaces and also experienced problems with incomplete sensor coverage and poor sensitivity [18]. Although this direct approach can provide detailed information on the location and force of a contact, it can be difficult to integrate with complex finger geometries, and is only capable of detecting contacts made on the instrumented area.

In comparison to these contact sensors, our approach has a detection threshold of between 0.03 and $0.8 \mathrm{~N}$ depending on the contact location. The minimum threshold of $0.03 \mathrm{~N}$, corresponding to contacts at the tip of the finger, is similar to the sensitivity of these other sensors. In addition, our sensor is capable of detecting contacts on any surface of the finger; something that the tendon tension or finger pad sensors are incapable of doing. Finally, although the six-axis load cell is also capable of detecting contacts anywhere on the finger tip, it is significantly larger and more difficult to integrate into a finger than the accelerometer required for our method, can easily be damaged, and is orders of magnitude more expensive. Also, while we cannot speculate as to the specifics, we have observed that many of the reported performance specs for available sensors are based on testing in a highly structured environment and the sensitivity in practice, after reasonable thresholds have been built in to avoid false positives due to mechanical noise and other effects, is much smaller than the reported values. All of these effects considered, our sensor is perhaps slightly lower in sensitivity than other available options, but is not limited to only sensorized surfaces, is extremely durable as it can be embedded within the structure, and is inexpensive and easy to implement.

\section{LIMITATIONS AND FUTURE WORK}

The approach to contact sensing that we present here is a lowcost solution capable of detecting contact on all faces of a finger with a low force threshold and reasonable time response. However, it does suffer from a number of limitations in addition to those already discussed that, when addressed, will significantly expand its applicability.

One limitation of the current prototype is that flexing the digit causes the resonant frequency of the digit to rise and register as a false contact. Therefore, as currently implemented with a fixed frequency threshold, the sensor should only be used when the hand is unactuated, such as during reaching phase of grasping. However, this limitation can be addressed by characterizing the free resonant frequency of the finger as a function of tendon position and implementing a tendon excursion dependent frequency threshold. This modification would allow the contact sensor to function as the fingers are closed during grasping.

Another limitation is that the excitation input is currently provided by the primary actuator. The current system uses a single back-drivable motor to both flex the finger and excite it. Although this can be beneficial in that an additional actuator is not needed for the system, it does impose a number of requirements on the motor, namely that it must be able to oscillate at the torque and frequency needed to excite the system at resonance. This requirement precludes the use of small low torque motors with large gear reductions. However, because the sensor operates independent of excitation method, it is possible to use two actuators, one optimized for flexion of the digit and the other such as a fast acting solenoid for excitation in applications where a single larger motor is impractical.

Finally, because our sensor relies on relatively low-frequency excitation of the digits, its operation might interfere with other sensors attached to the hand. For example, if our sensor is used in conjunction with an extrinsic contact sensor such as a load cell or piezoelectric film, the oscillation of the finger could be registered as noise by that sensor. However, this limitation may be addressed by switching between sensors; for example, the vibration-based sensor could be used until contact is detected, at which point it could be deactivated in favor of another sensor.

One aspect of the sensor that we have not systematically quantified yet is its performance in various orientations or under accelerations that we might expect to see during use on a manipulator attached to a robotic arm. Initial experimentation indicates that the system does not falsely detect contact when its orientation is changed or it undergoes low accelerations. However, future characterization should be performed under various operating conditions to determine what conditions such as cyclic oscillation of the sensor could cause it to fail.

Although the current system works well, it may be possible to further improve upon its performance by utilizing both accelerometers' signals concurrently. The time response of the system may be improved by detecting contact based on either of the accelerometers' signal's rising where false positives could be eliminated by waiting until both signals rise before detecting a contact. Finally, it may be possible to implement another approach such as measuring the phase difference between the two signals. This, in turn, could be used to further quantify measurements such as estimating the contact location.

\section{CONCLUSION}

This paper has presented a novel approach to contact sensing that uses low-cost integrated circuit components and the existing actuator and is capable of detecting contact anywhere on the finger. Unlike most other contact sensors, this approach did not involve instrumenting the surface of the digit with sensor arrays or the construction of a specialized finger, making it easy to implement with existing compliant finger designs. This sensor, using a frequency-based metric, was capable of detecting contacts at forces of 0.03 to $0.8 \mathrm{~N}$ depending on the contact 
location. This metric was compared to an amplitude-based metric that failed to detect some contacts entirely and had a force threshold of between 0.1 and $8 \mathrm{~N}$ depending on the contact location. Although the sensor performed well for contacts on all surfaces of the finger (including out-of-plane) the sensitivity is a function of the contact location, velocity, and stiffness. However, the authors are unaware of any other approach, aside from mounting the finger on a multiaxis force/torque sensor, that is able to detect contact on all surfaces of the finger without specifically instrumenting those surfaces, and particularly not with the relative simplicity of the approach proposed here.

\section{ACKNOWLEDGMENT}

The authors would like to thank L. Odhner and R. Howe for their helpful discussions related to this paper.

\section{REFERENCES}

[1] G. Westling and R. Johansson, "Factors influencing the force control during precision grip," Exp. Brain Res., vol. 53, pp. 277-284, 1984.

[2] J. Tegin and J. Wikander, "Tactile sensing in intelligent robotic manipulation-A review," Ind. Robot, Int. J., vol. 32, pp. 64-70, 2005.

[3] E. R. Kandel, J. H. Schwartz, and T. M. Jessell, Principles of Neural Science. New York, USA: McGraw-Hill, 2000.

[4] K. Motoo, F. Arai, and T. Fukuda, "Piezoelectric vibration-type tactile sensor using elasticity and viscosity change of structure," IEEE Sensors J., vol. 7, no. 7, pp. 1044-1051, Jul. 2007.

[5] S. Omata and Y. Terunuma, "New tactile sensor like the human hand and its applications," Sens. Actuators A, Phys., vol. 35, pp. 9-15, 1992.

[6] A. M. Dollar, L. P. Jentoft, J. H. Gao, and R. D. Howe, "Contact sensing and grasping performance of compliant hands," Auton. Robots, vol. 28, pp. 65-75, 2010.

[7] P. Yong-Lae, K. Chau, R. J. Black, and M. R. Cutkosky, "Force sensing robot fingers using embedded fiber bragg grating sensors and shape deposition manufacturing," in Proc. IEEE Int. Conf. Robot. Autom., 2007, pp. $1510-1516$.

[8] H. Maekawa, K. Tanie, and K. Komoriya, "A finger-shaped tactile sensor using an optical waveguide," in Proc. Int. Conf. Syst., Man Cybern., Syst. Eng. Serv. Humans, 1993, vol. 5, pp. 403-408.

[9] J. Butterfass, M. Grebenstein, H. Liu, and G. Hirzinger, "DLR-Hand II: Next generation of a dextrous robot hand," in Proc. IEEE Int. Conf. Robot. Autom., 2001, vol. 1, pp. 109-114.

[10] S. B. Backus and A. M. Dollar, "Robust, inexpensive resonant frequency based contact detection for robotic manipulators," in Proc. IEEE Int. Conf. Robot. Autom., St. Paul, MN, USA, 2012, pp. 1514-1519.

[11] A. M. Dollar and R. D. Howe, "A robust compliant grasper via shape deposition manufacturing," IEEE/ASME Trans. Mechatronics, vol. 11, no. 2, pp. 154-161, Apr. 2006.

[12] H. Yousef, M. Boukallel, and K. Althoefer, "Tactile sensing for dexterous in-hand manipulation in robotics-A review," Sens. Actuators A, Phys., vol. 167, pp. 171-187, Jun. 2011.
[13] R. D. Howe and M. R. Cutkosky, "Sensing skin acceleration for slip and texture perception," in Proc. IEEE Int. Conf. Robot. Autom., 1989, vol. 1, pp. $145-150$.

[14] C. Rudolf and W. Seemann, "Resonant excitation of a composite beam using piezoelectric MFC actuators," in Proc. Int. Conf. New Actuators, 2008.

[15] S. Omata, Y. Murayama, and C. E. Constantinou, "Real time robotic tactile sensor system for the determination of the physical properties of biomaterials," Sens. Actuators A, Phys., vol. 112, pp. 278-285, 1 May, 2004.

[16] S. Jacobsen, E. Iversen, D. Knutti, R. Johnson, and K. Biggers, "Design of the Utah/M.I.T. dextrous hand," in Proc. IEEE Int. Conf. Robot. Autom., 1986, pp. 1520-1532.

[17] B. T. Inc., "Barrett hand," BH8-280 Data Sheet, ed., 2011.

[18] T. B. Martin, R. O. Ambrose, M. A. Diftler, R. Platt, Jr., and M. J. Butzer, "Tactile gloves for autonomous grasping with the NASA/DARPA Robonaut," in Proc. IEEE Int. Conf. Robot. Autom., 2004, vol. 2, pp. 1713-1718.

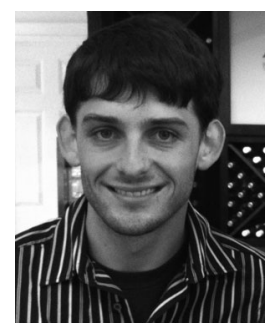

Spencer B. Backus (S'12) received the B.S. degree in mechanical engineering from F. W. Olin College of Engineering, Needham, MA, USA, in 2010, and the M.S. degree in mechanical engineering from Yale University, New Haven, CT, USA, in 2012, where he is currently working toward the Ph.D. degree in engineering.

His research interests include bioinspired design, underactuated manipulation, sensor development, and grasping from mobile robots.

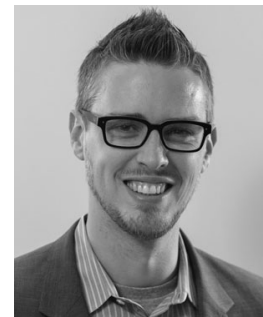

Aaron M. Dollar (M'06-SM'13) received the B.S degree in mechanical engineering from the University of Massachusetts, Amherst, MA, USA, in 2000, and the S.M. and Ph.D. degrees in engineering sciences from Harvard University, Cambridge, MA, USA, in 2006.

He conducted two years of postdoctoral research at the MIT Media Lab and currently is the John J. Lee Assistant Professor of Mechanical Engineering and Materials Science at Yale University, New Haven, CT, USA. His research topics include human and robotic grasping and dexterous manipulation, mechanisms and machine design, and assistive and rehabilitation devices including upper limb prosthetics and lower limb orthoses.

Dr. Dollar is a member of the American Society of Mechanical Engineers. He received the 2013 DARPA Young Faculty Award, the 2011 AFOSR Young Investigator Award, the 2010 Technology Review TR35 Young Innovator Award, and the 2010 NSF CAREER Award. 